Rev. Saúde puibl., S. Paulo

$11: 170-81, \quad 1977$

\title{
SITUAÇÃO VACINAL DA POPULAÇÃO DISCENTE DA UNIVERSIDADE DE SÃO PAULO
}

\author{
Ernesto Lima Gonçalves* \\ Gláucia Pereira Salles* \\ Sérgio Reis Quaglia**
}

RSPU-B/349

Gonçalves, E. L. et al. Situação vacinal da população discente da Universidade de São Paulo. Rev. Saúde públ., S. Paulo, 11: 170-81, 1977.

Resumo: São apresentados aspectos da situação vacinal dos alunos dos Institutos da Universidade de São Paulo, situados na capital e nos "campi" do interior em Ribeirão Preto, São Carlos, Baurll e Piracicaba, Estado de São Paulo, Brasil. Foi realizado inquérito preliminar mediante a aplicação de questionário especial a uma amostra de 13.139 alunos (42,3\% do total de matriculados), sendo 1.707 alunos no interior (38,3\% dos matriculados) e 11.432 na capital (43\% dos matriculados). Os principais dados sobre vaciną̧äo antivariólica foram: $20 \%$ dos alunos do interior e $30 \%$ dos da capital informaram não ter a cicatriz ou não saber de sua existência; $50 \%$ dos alunos do interior $e$ 75\% dos da capital informaram ter sido vacinados há menos de 5 anos; $35 \%$ do total de alunos afirmaram que o resultado da última vacina recebida foi positivo. Não foram vacinados contra o tétano ou não receberam a vacina de acordo com o esquema preconizado pela Secretaria de Estado da Saude, $2 / 3$ dos alunos. Os dados sobre vacinaçâa antimeningocócica revelaram que $17 \%$ dos alunos do interior e $80 \%$ dos da capital declaravam ter sido vacinados. Foram examinados 3.113 estudantes, definindo-se pela vacinação antivariólica de 405 deles (13\% do total); destes, $253(62,5 \%)$ retornaram para leitura, que foi positiva em 221 $(87,3)$ deles. Em relação à vacina antitetânica, 1.118 alunos da USP receberam as duas doses programadas, o que dá um indice de 66,9\%; considerados separadamente os alunos de graduação e de pós-graduação, esse indice foi respectivamente de $66,1 \%$ e $80,2 \%$.

UnITERmos: Universitários, estado vacinal. Vacinação. Medicina preventiva.

INTRODUÇA

A Universidade de São Paulo (USP) representa de longe a maior instituição universitária de nosso Estado, abrangendo um número de alunos equivalente à soma das Universidades: Mackenzie, de Campinas, Federal de São Carlos, Católicas de Campinas e de São Paulo. Em 1975 a USP abrigava um total de 31.014 estudantes de graduação, distribuídos pelos "campi" da capital (26.558), de Ribeirão Preto (1.903), de São Carlos (1.248), de Piracicaba (1.101) e de Bauru (204).

Dentro de sua estrutura administrativa, conta a USP com a Coordenadoria de Saúde e Assistência Social (COSEAS), na qual se insere uma Divisão de Saúde destinada a prestar serviços à população uni-

* Da Faculaade de Medicina da USP. Av. Dr. Arnaldo, 455, Sâo Paulo, SP - Brasil e da Divisão de Saúde da Coordenadoria da Saú de e Assistência Social (COSEAS) da USP Cidade Universitária - São Paulo, SP - Brasil.

* Da Divisão de Saúde da Coordenadoria de Saúle e Assistência Social da USP. 
GONÇALVES, E. L. et al. - Situação racinal da população discente da Universidade de São Paulo. Rev. Saúde públ., S. Paulo, 11:170-81, 1977.

versitária. Considerando a cobertura previdenciária de que já gozam professores e funcionários da Universidade, a ênfase tem sido colocada no atendimento dos alunos, tanto de graduação quanto de pós-graduação, incluindo seus dependentes. Saliente-se que todo atendimento é prestado de maneira gratuita, incluindo assistência ambulatorial, realização de exames complementares de diagnóstico e internação hospitalar para tratamento clínico, cirúrgico e atendimento obstétrico.

\section{SITUAÇÃO VACINAL}

Na Tabela 1 aparecem alguns dados estatísticus sobre 0 atendimento prestado aos estudantes da USP no ambulatório da Divisão de Saúde da COSEAS, situado na capital, na Cidade Universitária.

T A B E L A 1

Média mensal de atendimentos ambulatoriais prestados a estudantes da USP pela Divisão de Saúde da Coordenadoria de Saúde e Assistência Social da USP

\begin{tabular}{|c|c|c|c|c|c|c|}
\hline \multirow{2}{*}{$\begin{array}{c}\text { Tipo de } \\
\text { atendimento }\end{array}$} & \multicolumn{6}{|c|}{ Anos de funcionamento } \\
\hline & 1971 & 1972 & 1973 & 1974 & 1975 & $1976 *$ \\
\hline Consultas médicas & 1796 & 2249 & 2274 & 2327 & 2402 & 2937 \\
\hline Aplicações fisioterápicas & 115 & 137 & 159 & 214 & 397 & 350 \\
\hline Atendimentos psicológicos & 26 & 60 & 103 & 160 & 265 & 248 \\
\hline Atendimentos odontológicos & 401 & 643 & 870 & 729 & 1090 & 817 \\
\hline Atendimento enfermagem & 315 & 573 & 628 & 1678 & 4649 & 5204 \\
\hline $\mathbf{T} O \mathbf{T} \mathbf{A} \mathbf{L}$ & 2653 & 3632 & 4034 & 5108 & 8803 & 9556 \\
\hline
\end{tabular}

* Média dos valores do 10 semestre.

Fonte: Seção Controle e Análise de Dados da Divisão de Saúde da COSEAS - USP, 1976.

Os dados revelam um aumento considerável no número de atendimentos prestados, em particular nos últimos anos, o que se deve basicamente a um apreciável esforço de racionalização de atividades, uma vez que não houve qualquer aumento substancial no quadro profissional. De outro lado, como ao longo desses anos, não houve crescimento apreciável do número de alunos matriculados na USP, o aumento quantitativo dos atendimentos prestados representa evidentemente um melhor nivel assistencial prestado à população universitária.

Desde 1974, vem a Divisão de Saúde da COSEAS dedicando especial atenção a uma área de relevante importância no contexto da assistência médica de qualquer comunidade: trata-se dos aspectos ligados à medi- cina preventiva. Em princípios daquele ano, por portaria do Coordenador da COSEAS, foi criado um Grupo de Trabalho destinado a definir um "Plano de assistência à população da USP, referente aos aspectos preventivos". O Grupo terminou por preparar um programa extremamente vasto e até audacioso, que apenas ao longo do tempo poderá ser desenvolvido integralmente.

De todas as proposições contidas no referido Plano, a Divisão de Saúde optou por atribuir prioridade aos programas de imunização e de assistência materno-infantil. Como o objetivo deste trabalho é analisar a situação vacinal dos alunos da USP, deixaremos de lado os problemas da assistência materno-infantil. 
GONÇALVES, E. L. et a], - Situação vacinal da população discente da Universidade de São Paulo. Rev. Saude públ., S. Paulo, 11:170-81, 1977.

Nos anos anteriores, a atitude da Divisão de Saúde no campo da imunização permaneceu passiva, limitando-se a vacinar os interessados que compareciam ao ambulatório. Foram assim imunizados, nos anos de 1970, 1971, 1972 e 1973, respectivamente $352,101,108$ e 814 pessoas contra varíola e 256,179, 419 e 1850 contra tétano.

A partir de 1974, contudo, preferiu-se abordar o problema de maneira mais planejada: inicialmente desenvolveu-se um inquérito sobre a situação vacinal dos alunos da USP, tanto na capital quanto no interior. A amostra pesquisada no interior foi de 1.707 alunos ( $38,3 \%$ dos matriculados) e na capital chegou a 11.432 alunos (43\% dos matriculados).

A Tabela 2 apresenta os resultaclos relativos à vacinação antivariólica.

A respeito da existência de cicatriz, elemento físico bastante eviclente, chama a atenção o fato de que mais de $20 \%$ dos alunos do interior informaram não ter a cicatriz ou não saber de sua existência; entre os alunos da capital, esse numeru thegou a quase $30 \%$.

O prazo da ultima vacina mostrou apreciável diferença entre a capital - onde $75 \mathrm{r}$ dos alunos informaram ter sido vacinados há menos de 5 anos - e o interior. unde esse número é da ordem de $50 \%$. Em relação ao resultado da última vacina recebida, saliente-se que cerca de $35 \%$ dos alunos, tanto na capital quanto no interior, referiram que ele foi positivo, o que pode indicar baixa condição de imunização, justificando amplamente uma permanente preocupação com programas destinados a melhorar essa situação.

A Tabela 3 compreende os resultados do inquérito desenvolvido, no que se refere à vacina antitetânica.

Os dados relativos à vacinação antitetânica são muito menos animadores do que acpueles referentes à vacinação antivariólica, pois, nos fazem supor que cerca de $2 / 3$ dos alunos da USP não foram vacinados ou não receberam a vacina segundo esquema preconizado pela Secretaria de Estado da Saúde. Saliente-se que esses números são também elevados mesmo no caso de alunos altamente expostos, como é o caso dos que freqüentam escolas de Agronomia e de Zontecnia.

A Tabela 4 apresenta os resultados relativos à vacinação antimeningocócica.

Em relação à vacinação antimeningociocica, é indispensável esclarecer que a grande discrepancia de dados entre os alunos do interior e us da capital deriva do fato de que 0 inquérito foi realizado no interior, antes do grande programa de vacinação desenvolvido no $1^{\circ}$ semestre de 1975 , em todo (1) Estado de Sāo Paulo. Verifica-se, pelos dados da Tabela 4, que apenas cerca de $17 \%$ da população universitária (alunos do interior) estavam imunizados contra a meningite meningocócica, antes do desenvolvimento do referido programa de vacina. Depois da vacina em massa desenvolvida, pouco mais de $80 \%$ da pupulação universitária (amostra representada pelos alunos da capital) apresentava-se imunizada contra us tipos $\mathrm{A}$ e $\mathrm{C}$ do meningococo.

Com base nos dados obtidos pelo inquérito, foram elaborados programas de vacinação para os alunos da Universidade de São Paulo. Como é natural, seu desenvolvimento deveria contar com a cooperação indispensável dos órgãos da Secretaria de Estado da Saúde. Por razões ligadas às atividades da USP, optou-se pelo desenvolvimento de um programa voltado para os alunos dos institutos localizados no interior.

Segundo diretrizes da Secretaria da Saude, foram adotados os seguintes critérios de vacinação:

1. em relação à vacina antivariólica (VAV) - somente para os não portadores de cicatriz vacinal;

2. em relação à vacina antitetânica (VAT) - para aqueles que não completaram o esquema de vacinação ou não tinham sidn vacinados de acordo com o esquema preconizado pela Secretaria da Saúde. 


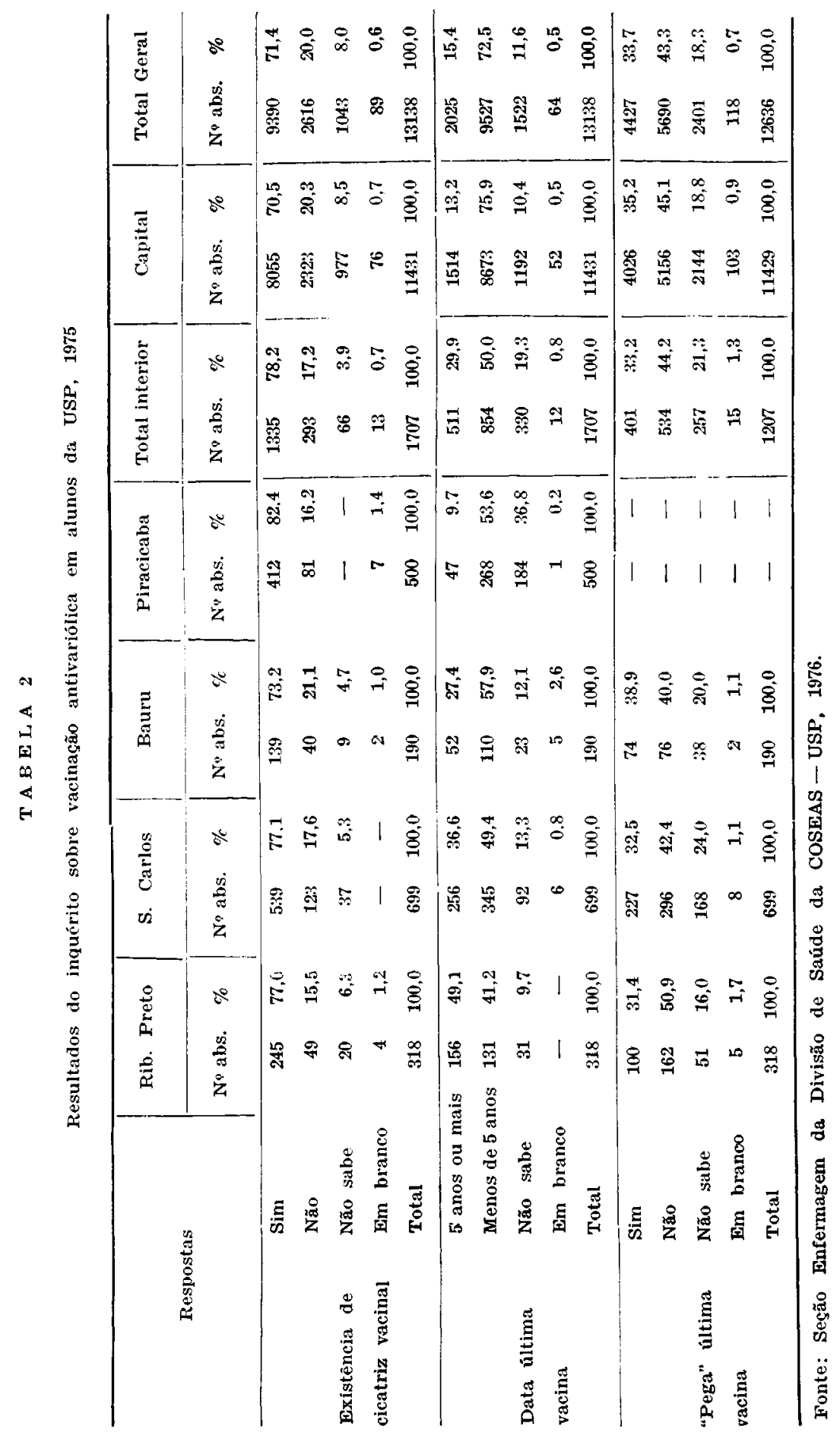




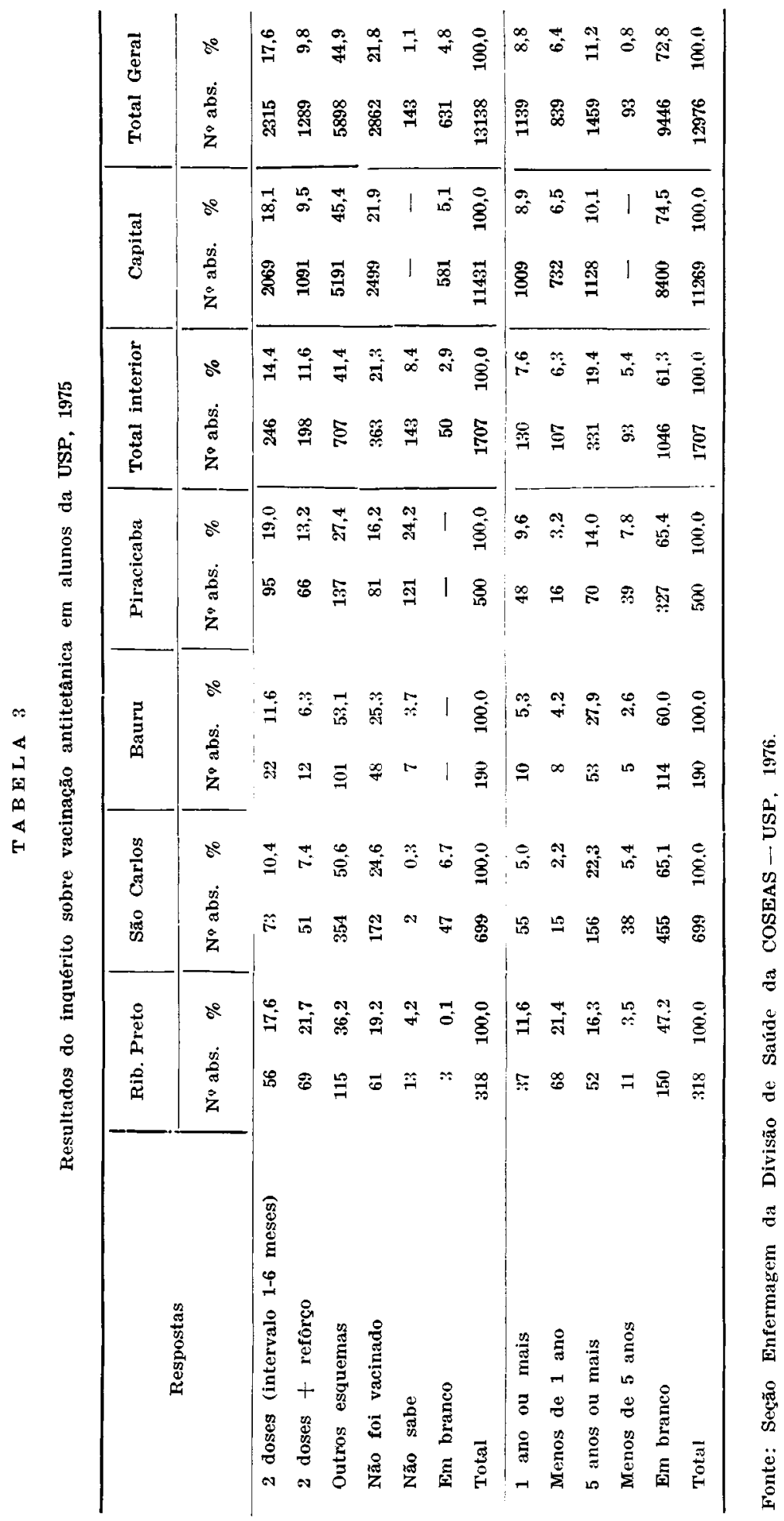


GONÇALVES, E. L. et al. - Situação vacinal da população discente da Universidade de São Paulo. Rev. Saúde publ., S. Paulo, 11:170-81, 1977.

TAB ELA 4

Resultado do inquérito sobre vacinação antimeningocócica em alunos da USP, 1975

\begin{tabular}{|c|c|c|c|c|c|c|c|}
\hline Localização & Valor & Tipo A & Tipo $\mathrm{C}$ & $\begin{array}{r}\text { Vacina } \\
A+C\end{array}$ & recebida & Em branco & Total \\
\hline Ribeirão Preto & $\begin{array}{c}\text { Absol. } \\
\%\end{array}$ & $\begin{array}{r}7 \\
2: 2\end{array}$ & $\begin{array}{r}6 \\
1,9\end{array}$ & $\begin{array}{c}21 \\
6,6\end{array}$ & $\begin{array}{r}268 \\
84,3\end{array}$ & $\begin{array}{l}16 \\
5,0\end{array}$ & $\begin{array}{c}319 \\
100,0\end{array}$ \\
\hline São Carlos & $\begin{array}{c}\text { Absol. } \\
\%\end{array}$ & $\begin{array}{l}31 \\
4,4\end{array}$ & $\begin{array}{c}10 \\
1,4\end{array}$ & $\begin{array}{r}78 \\
11,2\end{array}$ & $\begin{array}{c}55: 3 \\
79.1\end{array}$ & $\begin{array}{r}27 \\
3.9\end{array}$ & $\begin{array}{c}699 \\
100,0\end{array}$ \\
\hline Bauru & $\begin{array}{c}\text { Absol. } \\
\%\end{array}$ & $\begin{array}{l}12 \\
6,2\end{array}$ & $\begin{array}{c}5 \\
2,6\end{array}$ & $\begin{array}{c}44 \\
23,2\end{array}$ & $\begin{array}{l}125 \\
65,8\end{array}$ & $\begin{array}{r}4 \\
2,1\end{array}$ & $\begin{array}{c}190 \\
100.0\end{array}$ \\
\hline Piracicaba & $\begin{array}{c}\text { Absol. } \\
\%\end{array}$ & - & - & - & - & - & - \\
\hline Total interior & $\begin{array}{c}\text { Absol. } \\
\%\end{array}$ & $\begin{array}{l}50 \\
4,1\end{array}$ & $\begin{array}{l}21 \\
1,7\end{array}$ & $\begin{array}{r}143 \\
11,8\end{array}$ & $\begin{array}{r}946 \\
78,5\end{array}$ & $\begin{array}{l}47 \\
3,9\end{array}$ & $\begin{array}{l}1207 \\
100,0\end{array}$ \\
\hline Capital & $\begin{array}{c}\text { Absol. } \\
\%\end{array}$ & $\begin{array}{c}646 \\
5,7\end{array}$ & $\begin{array}{c}711 \\
6,2\end{array}$ & $\begin{array}{r}9273 \\
81,1\end{array}$ & $\begin{array}{r}445 \\
3,9\end{array}$ & $\begin{array}{r}355 \\
3,1\end{array}$ & $\begin{array}{r}11430 \\
100,0\end{array}$ \\
\hline Total geral & $\begin{array}{c}\text { Absol. } \\
\%\end{array}$ & $\begin{array}{r}696 \\
5,5\end{array}$ & $\begin{array}{r}732 \\
5,6\end{array}$ & $\begin{array}{r}9416 \\
74,5\end{array}$ & $\begin{array}{r}1391 \\
11,0\end{array}$ & $\begin{array}{r}402 \\
3,2\end{array}$ & $\begin{array}{r}12637 \\
100,0\end{array}$ \\
\hline
\end{tabular}

Fonte: Seção Enfermagem da Divisão de Saúde da COSEAS - USP, 1976.

O programa previa duas etapas, com intervalos de 30 dias, destinadas à aplicação das duas doses de vacina antitetânica e à verificação dos resultados da vacinação antivariólica. Em ambas adotou-se a seguinte sequiência de operação: 1. Identificação do cliente; 2 . conferência da cicatriz de VAV (por enfermeira, atendente do Distrito Sanitário e/ou visitadora sanitária); 3 . obtenção de informações sobre doses de VAT recebidas anteriormente, com conferência de possiveis contra-indicações; 4 . anotação e fornecimento da caderneta; 5 . aplicação da vacina; 6 . recomendação de retorno para avaliação; 7. prestação de outros esclarecimentos solicitados.

$\mathrm{Na}$ segunda etapa, procedeu-se à avaliação dos resultados da VAV aplicada no mês anterior, recomendando-se àqueles que recebiam a VAT, como 1a dose, a continuação da vacinaçăo por meio dos Centros de Saúde estaduais.

Definidos os critérios e as diretrizes, 0 programa foi desenvolvido nos meses de outubro e novembro de 1975 , compreendendo os Institutos da USP situados em Ribeirão Preto, São Carlos, Piracicaba e Bauru. Embora a iniciativa visasse essencialmente os alunos, a vacinação foi estendida também a funcionários e professores. $\mathrm{Na}$ Tabela 5 aparecem os totais de alunos, professores e funcionários de cada "campus" da USP no interior.

Nas Tabelas 6 e 7 figuram os dados relativos às duas etapas do programa desenvolvido; os percentuais incluídos nestas tabelas referem-se aos totais da Tabela 5 .

No que se refere à vacina antivariólica, a possibilidade da conferência da cicatriz vacinal permitiu-nos obter uma amostragem 
GONÇALVES, E. L. et al. - Situação vacinal da população discente da Universidade de São Paulo. Rev. Saúde públ., S. Paulo, 11:170-81, 1977.

\begin{tabular}{|c|c|c|c|c|}
\hline \multicolumn{4}{|c|}{ TABELA 5} & $\cdots$ \\
\hline \multirow{2}{*}{ "Campi" e Institutos } & \multicolumn{2}{|c|}{ Alunos } & \multirow{2}{*}{$\begin{array}{l}\text { Profesosres e } \\
\text { funcionários }\end{array}$} & \multirow{2}{*}{ Total } \\
\hline & Graduação & Pós-graduação & & \\
\hline \multicolumn{5}{|l|}{ Ribeirăo Preto } \\
\hline Fac. Medicina & 631 & 356 & 633 & 1620 \\
\hline Esc. Enfermagem & 162 & 20 & 80 & 262 \\
\hline Fac. Filosofia & 585 & - & 145 & 730 \\
\hline Fac. Farm. Odontologia & 543 & - & 206 & 740 \\
\hline Subtotal & 1912 & 376 & 1064 & 3352 \\
\hline São Carlos & 1142 & 500 & 570 & 1762 \\
\hline Bauru & 204 & 30 & 250 & 484 \\
\hline Piracicaba & 1101 & 360 & 880 & 2341 \\
\hline Total geral & 4359 & 1266 & 2764 & 8389 \\
\hline
\end{tabular}

Fonte: Seção Enfermagem da Divisão de Saúde da COSEAS - USP, 1976.

da situação em população adulta. Assim, na primeira etapa, para uma população universitária de 1.806 alunos examinados, foram aplicadas 220 doses de VAV; este número corresponde a $12,2 \%$ daquele total, correspondendo às pessoas em que não foi encontrada cicatriz ou em que havia dúvida. Esse percentual não difere muito dos $21,9 \%$ identificados pelo inquérito, conforme se verifica na Tabela 2. Esses números ficam ainda mais próximos, quando se considera que, na segunda etapa, entre 2.465 examinados, foi possivel ainda identificar mais 57 casos considerados como primovacinações.

As percentagens de conferência ficaram na dependência de comparecimento voluntário dos que haviam recebido a vacina antivariólica na primeira etapa. Não se processou a conferência de reações positivas à VAV aplicada na segunda etapa.

Alguns dados devem ser salientados. Assim, em Piracicaba, para um total de 165 doses de VAV, aplicadas inicialmente, houve o retorno de 124 pessoas $(\mathbf{7 5 , 1 \% )}$, verifi- cando-se 113 reações positivas, com um indice de $91,1 \%$ de positividade. Em Bauru, para 61 doses aplicadas na primeira etapa, houve o retorno para conferência de 47 pessoas $(77,1 \%)$, com 34 reações positivas, do que resulta um indice de $72,3 \%$ de positividade. Em Ribeirão Preto, para o total de 179 doses aplicadas, houve retorno para leitura de 82 pessoas $(45,8 \%)$ com 74 reações positivas $(90,2 \%$ de positividade).

Os dados parecem justificar a vacinação antivariólica ministrada. Recordando que a Campanha de Erradicação da Variola divulgou o alcance de $90,7 \%$ de pessoas vacinadas em 1970 em todo o Estado de São Paulo, concluimos pela conveniência da revacinação, considerando-se os índices de positividade obtidos.

Em relação à vacina antitetânica, durante a entrevista prévia para a triagem dos casos, foi possivel verificar que a maioria dos alunos não tinha realmente recebido esquema completo de vacinação ou nunca fora vacinada. Aqueles que já haviam recebido a vacina alguma vez năo sabiam infor- 


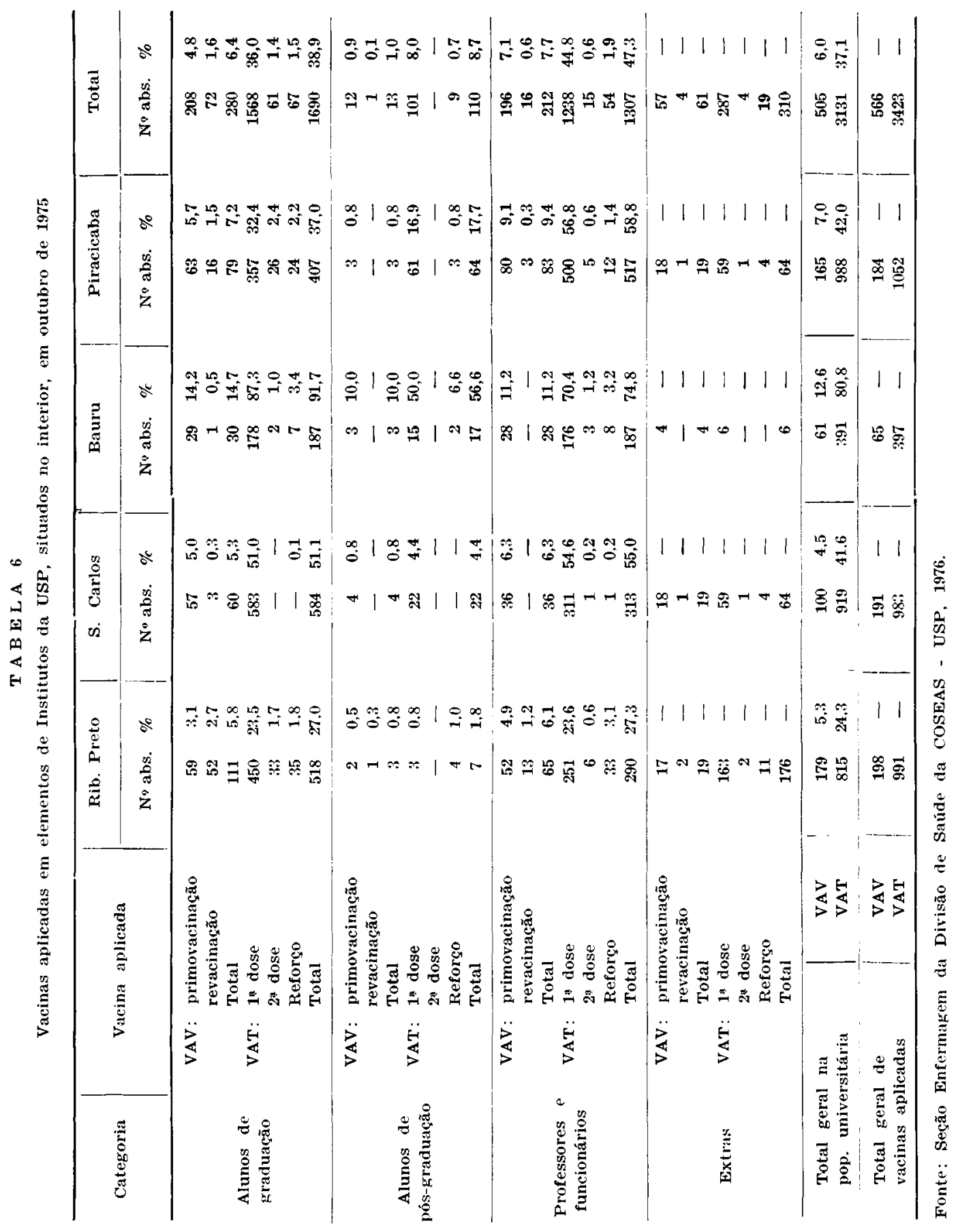




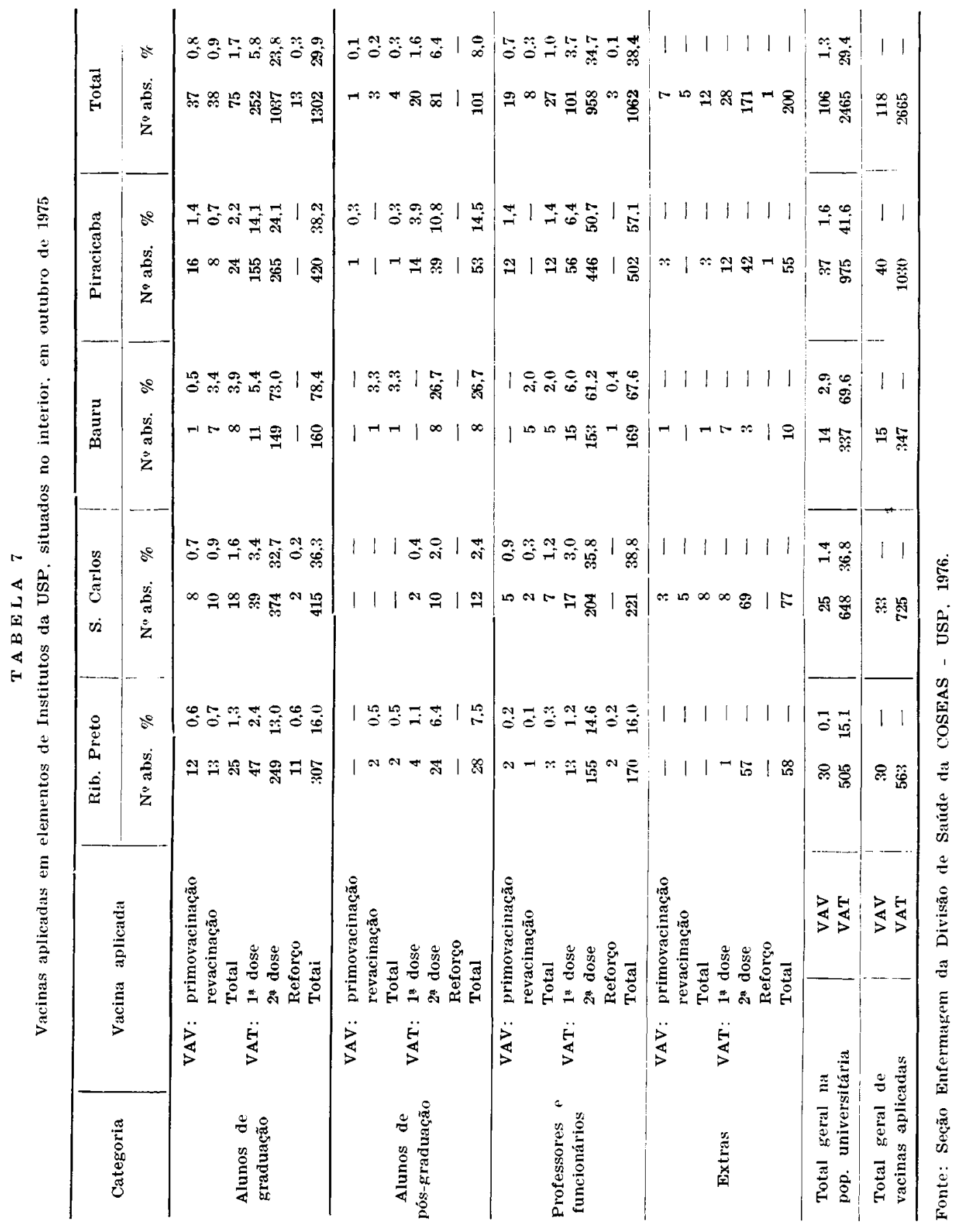


mar quando isso tinha ocorrido e muito poucos tinham algum comprovante de dose de VAT recebida anteriormente.

Tomando-se como base os clientes que voltaram na segunda etapa, para receber a segunda dose de VAT, conforme programado, pode-se estabelecer os seguintes percentuais de alunos que completaram a vacinação: em São Carlos, $64,1 \%$ dos alunos de graduação e $45,5 \%$ dos de pós-graduação; em Bauru, $83,7 \%$ dos alunos de graduação; em Piracicaba, $74,2 \%$ e $63,9 \%$ respectivamente dos alunos de graduação e de pós-graduação; em Ribeirão Preto, 55,3\% dos alunos de graduação. Nesta cidade, o número de segundas doses aplicadas em alunos de pós-graduação excedeu ao de primeiras doses registradas, pois, numero apreciável de alunos dessa categoria apresentou, na segunda etapa, comprovante de aplica(̧ão da dose anterior, fornecido pelo Hospital das Clinicas local, permitindo que o esquema de vacinação fosse completado.

Enı relação ao total da população universitária discente abordada, chegou-se à aplicação da segunda dose em $67,0 \%$ dos clientes que tinham recebido a dose inicial do anatox-tetânico.

Numerosas dificuldades tiveram que ser enfrentadas durante o desenvolvimentis da programação definida: insuficiência de divulgação educativa; distância geográfica impossibilitando um trabalho mais constante ou direto de motivação por parte da COSEAS, na Divisão de Saude; pioneirismo de uma iniciativa destinada especificamente à população universitária; aplicação de vacinas consideradas "de rotina", em relação às quais a população em geral manifesta pouco interesse.

Analisados os resultados, à luz dessas dificuldades, eles podem ser considerados satisfatórios, justificando a continuidade da iniciativa.

Em relação ao inquérito e levantamento de dados, as perguntas utilizadas para a obtenção das informações que figuram nas Tabelas 2,3 e 4 foram incorporadas, a partir de 1976, ao "Questionário Médico-
Esportivo", que todos us anos é preenchido pelos alunos ingressantes da USP. Dessa maneira, os dados obtidos em 1975 poderão ser permanentemente atualizados, obtendose uma avaliação dinâmica, ao longo do tempo, da situação vacinal de uma amostra considerável de população adulta em São Paulo. Pretende-se que ainda no corrente ano de 1976 seja realizada a última etapa de aplicação de VAT nos alunos do interior vacinados em 1975, bem como planejado " programa de imunização para os alunos da capital.

$$
\text { COMENTARIOS }
$$

$\dot{E}$ importante considerar que us dados epidemiológicos mais recentes, relativos à variola, mostram que a situação neste campo está bastante bem controlada. Preocupa muito mais o problema representado pelo tétano; várias razóes justifican essa preocupação.

Trata-se de uma doença que ainda apresenta apreciável indice de mortalidade; morrem anualmente cerca de meio milhão de individuos por tétano em todo " mundo, sendo yue, na America Latina, "Brasil lidera "s ubituário por tétano, em numeros absolutos. Basta dizer que se estima em 13.000 o numero de mortes anuais por essa doença, o çue dá um coeficiente de mortalidade da urdem de 13:100.000 habitantes; para efeitos de comparação, é útil lembrar que, nos países desenvolvidos ou naqueles cuịa população recebeu vacinação em massa, esse coeficiente tem oscilado entre 0,08 e 1,00:100.000 habitantes '. Em nosso meio, recentemente Barone e col. : referiram $20,6 \%$ de óbitos, num total de 461 doentes de tétano não unbelical. Deste número, 207 casos $(44 \%)$ estavam na faixa etária dominante nos integrantes do corpo discente da Universidade de São Paulo. Outros autores" têm encontrado indices de mortalidade ainda mais elevados de até $48 \%$, mesmo não incluindo casos de tétano neo-natal, seguramente mais graves. 
GONÇALVES, E. L. et al, - Situação vacinal da população discente da Universidade de São Paulo. Rev. Saude publ., S. Paulo, 11:170-81, 1977.

Barone e col. 2, estudando os casos de tétano do Hospital das Clínicas da Faculdade de Medicina da USP entre 1944 e 1969, verificaram um nitido deslocamento da incidência da doença para grupos etários mais avançados. Este fato é atribuído à vacinação compulsória da maior parte da população mais jovem, passando a doença a incidir predominantemente em indivíduos que não foram alcançados pelas campanhas de vacinação. É o que aconteceu com 343 casos $(74 \%)$ da série referida; apenas $14 \%$ tinham sido vacinados anteriormente.

No caso da amostra de alunos universitários de que estamos tratando, apenas cerca de $27 \%$ tinham recebido a vacina antitetânica, num esquema julgado satisfatório por nossas autoridades sanitárias. Esse percentual é bastante aproximado dos $33 \%$ referidos por Baldy e col. ${ }^{1}$, entre estudantes universitários da cidade de Londrina. A percentagem relativamente pequena de universitários vacinados contra o tétano é contrastante com o fato de que, na mesma amostra de Baldy e col. ${ }^{1}, 99,6 \%$ dos entrevistados conheciam a doença e $98,4 \%$ sabiam que a forma de transmissão no adulto é basicamente a partir de ferimentos contaminados. Na mesma amostra, 97,0\% sabiam da existência da vacina antitetânica e $93,8 \%$ reconheciam que a mesma era dotada de efeito preventivo. Uma das razões do baixo percentual de alunos vacinados talvez resida no fato de que muita gente atribui ainda pequena gravidade ao tétano; basta dizer que na amostra de Baldy e col. ${ }^{1} 42,4 \%$ dos universitários entrevistados consideravam pouco freqüente a mortalidade devida ao tétano.

Trata-se, evidentemente, de considerável desinformação a respeito do tétano. Basta lembrar que entre nós, em termos de morbidade, tem-se observado na casuistica do Hospital das Clínicas da Faculdade de Medicina da USP mais casos de tétano por ano do que os comprovados anualmente nos seguintes paises, todos juntos: Noruega, Inglaterra, Dinamarca, Suiça, Holanda, Austrália e Canadá ${ }^{6}$. Em termos de letali- dade, no interior do Estado de São Paulo, o tétano sozinho tem matado mais que onze importantes doenças infecciosas reunidas: difteria, coqueluche, sarampo, malária, meningite meningocócica, poliomielite e polioencefalite agudas, encefalites infecciosas, raiva, escarlatina, erisipela e desinteria amebiana 5. Recentemente, Fischmann e col." desenvolveram uma investigação epidemiológica do tétano no Rio Grande do Sul; examinando 136 fichas da Secretaria da Saúde daquele Estado, encontraram un indice de letalidade de $52,2 \%$ do total da amostra.

No caso da nossa amostra, representada por alunos da USP, a maior preocupação volta-se para os alunos da Faculdade Superior de Agricultura "Luiz de Queiroz", onde apenas $32 \%$ podem ser considerados efetivamente vacinados contra o tétano; trata-se de alunos que, por força do próprio tipo de aprendizado que recebem, desenvolvem apreciável número de atividades no campo. E fato sobejamente conhecido que o tubo digestivo dos herbivoros é o principal reservatório de bacilos do tétano e que o solo das regiōes onde abundam esses animais é altamente contaminado por esporos tetâni$\cos { }^{6}$. Esse fato explica provavelmente 0 achado de Fischmann e col. 3, que procurando relacionar o ferimento que causou a infecçāo com as atividades profissionais, verificaram que en $54 \%$ dos casos, em que se pôde identificar claramente a profissāo, o ferimento se dera durante essas atividades. No caso dos agricultores, contudo, esse indice chegava a $90,5 \%$.

Diante dos dados apresentados quanto à incidência e à mortalidade da infecção tetânica e da apreciável eficiência da vacinação respectiva ${ }^{3,4, \tau}$, justifica-se plenamente o esforço que vem sendo desenvolvido no âmbito da Universidade de São Paulo.

Deixando de lado os aspectos meramente médicos do problema, a importância econômica é relevante: com a quantia despen- 
GONÇALVES, E. L. et al. - Situação vacinal da população discente da Universidade de São Paulo. Rev. Saúde públ., S. Paulo, 11:170-81, 1977.

dida anualmente com o tratamento do tétano no Brasil seria possível vacinar cada ano, com duas doses de toxóide, mais 50 milhões de individuos ${ }^{6}$; ao lado de poupar-lhes a vida, seria possível liberar apreciável número de leitos hospitalares para o tratamento de doenças não previsíveis.
Mesmo em termos de tétano umbelical, existem indicações de que a vacinação das gestantes no último trimestre da gravidez é medida altamente aconselhável ${ }^{4}$; trata-se de iniciativa que já está sendo estudada, dentro do quadro da assistência pré-natal atualmente prestada a alunas da USP.

RSPU-B/:349

GONÇALVES, E. L. et al /Vaccinal condition of the student population of the $S$. Paulo University]. Rev. Saúde públ., S. Paulo, 11:170-81, 1977.

ABSTRACT: Some aspects regarding the vaccinal situation of students in the various "campi" of the São Paulo University (USP) are presented. At first an inquest was made, by means of a questionnaire which was answered by 13.139 people (42.3\% of the total university population). With respect to amallpox immunization, $20 \%$ of pupils from upcountry and $30 \%$ from São Paulo said they had no vaccination scar; $50 \%$ of pupils from upcountry and $75 \%$, from Säo Paulo said they were vaccinated about 5 years ago and $35 \%$ of all of them said that the last vaccination was efficient. About $2 / 3$ of all USP students were not vaccinated against tetanus or did not receive a recognized scheme of immunization. In reference to antimeningococcus immunization, the inquest was done in an upcountry "campi" before the wide official programme of May 1975; in the São Paulo "campus", the inquest was applied after this event. Because of this, only" $17 \%$ of hinterland people were said to be already immunized, as against $80 \%$ from São Paulo university students. The chosen patterns were absence of smallpox-vaccination scar and utilization of a non-recognized scheme of antitetanic vaccination. From 3.113 people examined, 405 received smallpox-vaccine, with $87.3 \%$ positive results. As regards antitetanic immunization, $67 \%$ of all examined people received the previously approved anatox dose.

UNITERMS: University population, vaccinal situation. Vaccination. Preventive medicine.

\section{REFERENCIAS BIBLIOGRAFICAS}

1. BALDY, J. L. S. et al. Tétano e vacinação antitetánica: estudo na população urbana de Londrina (PR), Brasli. Rev. Saúde públ., S. Paulo, 10:151-66, 1976.

2. BARONE, A. A. et al. Tétano: aspectos epidemiológicos, clínicos e terapêuticos: análise de 461 casos. Rev. Hosp. Clin. Fac. Med. S. Paulo, 31 :215-225, 1976.

3. FISCHMANN, $A$. et al. Investigação epidemiológica do tétano no Rio Grande do Sul, Brasil. Rev. Sauide públ., S. Paulo, 10:135-42, 1976.

4. LACAZ, C. S. - O problema do tétano no Bras. Rev. Ass, méd. bras., $12: 33-41$, 1966.
5. VERONESI, R. Tétano: grave problema de saúde pública no Brasil, Rev. Ass. paul Med., 52:456-60, 1958.

6. VERONESI, R. Tétano: um problema de saúde pública ainda não resolvido no Brasil. Rev. Ass. méd. bras., 12: 119-24, 1966.

7. VERONESI, $R$. Controle do tétano no Estado de São Paulo: análise e comentários em torno dos resultados da vacinação compulsiva dos escolares. Rev. Hosp. Clin. Fac. Med. S. Paulo, $26: 223-8$, 1971.

Recebido para publicação em 02/07/1976 Aprovado para publicaşão em 17/12/1976. 